\title{
Biomedical applications of targeted drug delivery, nanotheranostic and nuclear
}

\section{Medicine}

Idris zubairu sadiq ${ }^{1}$, Fatima Sadiq Abubakar ${ }^{1,2}{ }^{*} \operatorname{Hassan}_{\text {Aliyu Hassan }}{ }^{2}$,

${ }^{1}$ Department of Biochemistry, Faculty of life sciences, Ahmadu Bello University, Zaria-Nigeria

${ }^{2}$ National Agricultural Extension and Liaison Services, Ahmadu Bello University, Zaria-Nigeria

${ }^{3}$ Department of Biochemistry, Faculty of sciences Federal University, Dutse-Jigawa State

*izsadiq@abu.edu.ng

Abstract

Background: Convectional methods for drug delivery often faces setbacks due to systemic distribution, short half-life and degradation of therapeutics and therefore reduce concentrations of drug available to target tissue. Nanotheranostic provide a novel method for treating and diagnosing diseases

Methodology: collection and review of relevant literature

Result: while nanotheranostic offer advantage of personalized medicine and often combines diagnosis and therapy using single molecular approach, nuclear medicine relies on radioactive isotopes to diagnosed and destroys cancer cells. In both cases, nanocarriers such as lipid-based, polymer-based, drug-conjugate, inorganic 
nanoparticles are used to deliver drugs/probes/isotopes to target site, generating images and thereafter chemotherapy/radiotherapy begins.

Conclusion: Nanotheranostic plays important role in diseases diagnostic, therapy, imaging, monitoring of disease progression / response through the use of nanocarriers. This is made possible through nanoparticles/nanocarriers that delivers drug to the target tissues/cells.

Keywords: Nanocarriers, Nanotheranostics, Nuclear Medicine, Pharmacogenomics

\section{Introduction}

Nanotheranostic involves the use of nanoparticles mostly for diagnosis/therapy purposes for tracing biological pathways/metabolic reactions in order to acquire images for the purpose of diagnostic and therapy of diseases with the aim of establishing personalized medicine through the use of nanotechnology and nanomedicine $[1,2,3]$. A combination of diagnostic images and therapy are normally directed to the patients to provide tailored therapeutics. Sources of diagnostic images could be from ultrasound, Magnetic Resonance Imaging (MRI), nuclear medicine and computerized tomography scan (CT). In Nanomedicine, nanotechnological procedures such as nanobiocompatibles, nanorobots are applied in the field of medicine to diagnosed, treat, and prevent disease. Drugs are normally delivered to target tissue or organ via drug carriers such microspheres, liposomes, polymeric micelles and immune cells such as dendtritic cell, macrophages, neutrophils, dendritic cells, T lymphocytes, and natural killer cells [4]. Compared to the conventional method, nanotheranostic offer advantage of personalized medicine and often combines diagnosis and therapy using single molecular approach. Nanocarriers often deliver drug directly to the target, which facilitated action of the drugs and minimizes 
systemic transport, thus protect drug from degradation by indigenous enzymes and maintain bioavailability

\section{Nanotheranostics and nuclear medicine: the interrelationship}

Nuclear medicine is a branch of radiology that examines organ structure and functions using extremely small quantities of radioactive materials, or radiopharmaceuticals. While nanotheranostic provide a concept of using one molecule for both diagnosis and therapy using nanoparticles, nuclear medicine uses radioactive nuclides to evaluate the distribution of radioactive molecules through the use of PET of SPECT systems to acquire images and diagnose diseases or evaluate response to treatments $[5,6]$, and this is an especially applicable in the case of cancer diagnostic and therapy. It makes uses of curative radiations alpha and beta particles [7]. Gamma radiations permit the generation of investigative images (fig. 1) in monitoring drug distribution allowing extrapolation of dose reaching the target organ in relation to the whole body [8]. Radioisotopes have revolutionized nuclear medicine for example, phosphorus-32 and iodine-131 open doors for development of many radiopharmaceuticals including monoclonal antibodies $[9,10]$ and other biological probes. There are two phases of nanotheranostics; phase 1 (diagnostic phase) and Phase 2 (therapeutic phase). Phase 1 involves the diagnosis of a disease using radiopharmaceuticals that resemble substrate to the target tissue and thereafter imaging is done to diagnose the disease. In phase 2 , nanomedical strategies are used to deliver therapeutics/radiation to the target tissue (fig. 1). 


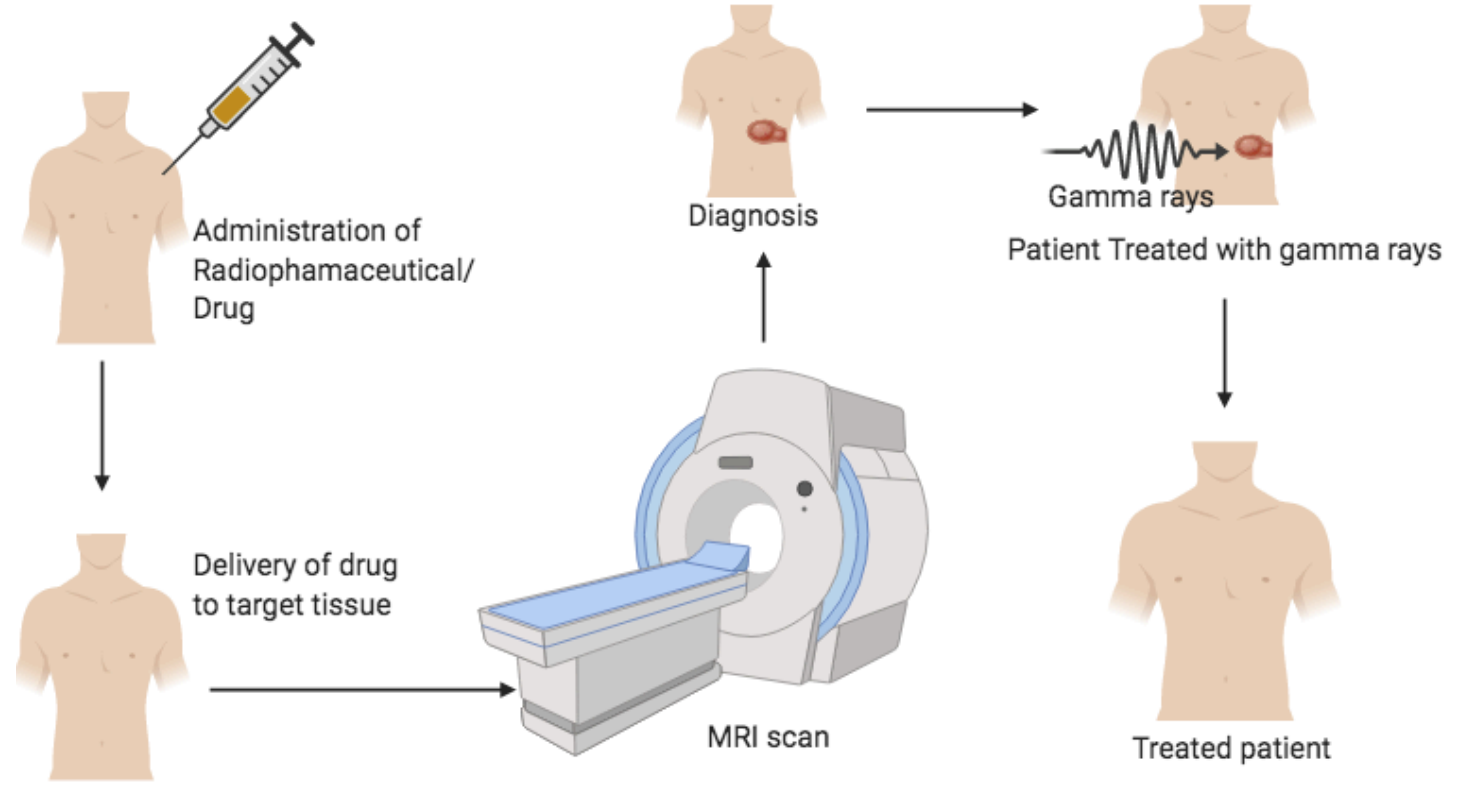

Figure1: Nanotheranostic agents in diagnostic, imaging and therapy

\section{Advantages of nanotheranostics in disease management and therapy}

- Discovery of new biological targets

- Monitoring disease progression/drug response

- Predicting systemic side effects

- Producing site specific pharmaceuticals

- Enhancing therapeutics response

- Provide avenue for personalize medicine

\section{Types of Ionizing radiations}

Alpha particles

Beta particles

Gamma rays

X-rays [11] 


\section{Nanotheranostic agents}

Nanotheranostic agents essentially consist of nanoparticles which play role in drug deliver, imaging, diagnosis and monitoring of therapeutic response. They are promising in the field of nanomedicine due to their ability to deliver substances to target sites. The table below listed some nanotheranostic agents [12].

Table 1: Nanotheranostic agent and their uses

\begin{tabular}{||l|l|l||}
\hline Nanotheranostic agent & Size & Uses \\
\hline Iron oxide Nanoparticle & $<20 \mathrm{~nm}$ & MRI imaging, Drug \\
Quantum dot & $2-10 \mathrm{~nm}$ & Imaging, labeling and \\
\hline Nanoparticle & & sensing \\
\hline Gold Nanoparticle & $10 \mathrm{~nm}$ & Drug conjugation \\
\hline \hline Carbon nanotubule & $0.4-40 \mathrm{~nm}$ & Drug delivery \\
\hline Nanoparticle & & Drug delivery, theranostic \\
\hline Silica Nanoparticle & $200 \mathrm{~nm}$ & purposes \\
\hline
\end{tabular}

Nanoparticles mediated-drug delivery in diagnosis and therapy

\section{Lipid base nanoparticles}

Lipid base nanocarriers are lipid vesicles used to transport both lipophilic and hydrophilic drug to their target. Example of lipid base nanocarriers include liposomes, bilayers and micelles etc. Many such nanomaterials can carry both hydrophobic and hydrophilic molecules, have quite minimal with no toxic effect, and extend the 
duration of therapeutic response by having a longer half-life and regulated drug release.

Advantages

- Less toxic [13]

- Deliver large amount of compound to their target site

- Form stable encapsulation of drugs [13]

- Antibodies could be attached to it $[14,15]$

- Transport both lipophilic and hydrophilic

\section{Polymer-based nanocarrirers}

Natural polymer base that are currently use in clinical settings include protein, peptides and albumin bound paclitaxel used in treatment of breast cancer [13]. Due to their versatility, synthetic polymers are promising tools for drug delivery [16]. An example of synthetic polymer base nanoparticle is PEG-PGA polymeric micelle [13]. Advantages

- Very effective in control drug release [16]

- Less toxic compared to the drug alone [16]

- Less side effect

- Stable polymer that are biodegradable [16]

- Increase solubility of drugs [13]

\section{Inorganic nanoparticles}

Inorganic nanoparticles are used in clinical and pharmacological settings to enhance radiotherapy, imaging and drug delivery (Fig.2)[17]. Examples include iron oxide, silica and gold nanoparticles.

Advantages

- Use for imaging [17]

- Used as Radiosensitiser e.g. iron oxide [18]

- Suitable for diagnostics [19] 
- Suitable Drug delivery [20]

\section{Drug conjugates}

Drug conjugates involve linking active pharmaceutical products to antibodies, peptide, aptamer, affibody or protein for targeted drug delivery and controlled released [21,22]. An example of drug conjugate is Trastuzumab emtansine used for treating breast cancer.

Advantages

- Improve targeted drug delivery

- Increase antitumor activities of cancer drugs [13]

- Less toxic and high efficacy

\section{Viral nanoparticles}

Viruses such as tumor-homing horses are engineered to produce therapeutics proteins that may be beneficial to cancer patients [13]. Poxviruses essentially uses characteristic of cancer cell to replicate in tumor cell thereby destroying the cell through activation of EGFR-Ras-MAPK signaling pathway [23].

\section{Advantages}

- Increase immunological resistance of the body to tumor [13]

- Destroy cancer cell via activation of EGFR-Ras-MAPK signaling pathway.
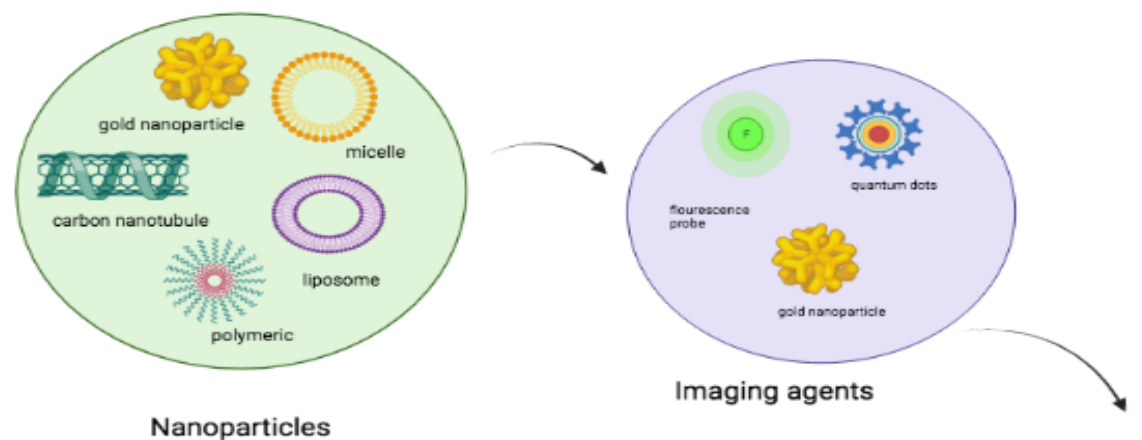

Nanoparticles

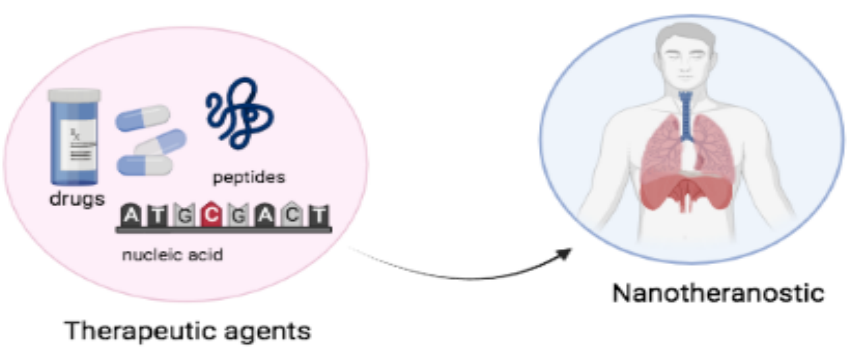

Figure 2: Nanotheranostic approach 


\section{Engineering Leukocytes to delivered drugs}

Leukocytes refer to the cells belonging to the immune system protecting the body against invaders and infectious disease. They are derived from hematopoietic stem cells and circulate round the body and recruited to tumor microenvironment and inflammation sites. Leukocytes include polymorphonuclear leukocytes (neutrophils, eosinophil, basophils and mast cells), lymphocytes (natural killer cells, T cells and B cells) and monocytes [24]. Delivery of leukocytes mediated nanoparticle involve packing of nanoparticles into leukocyte for example, macrophage (fig.3). It is then internalized rapidly through endocytosis via Fc $\gamma$ receptor [25]. Physical method such as electroporation may be use to enhanced nanoparticles uptake. Particles may also be attached to the surface of leukocytes provided it binding function is sustaining [24, $26,27]$. In in-vivo method, nanoparcticles are injected into the animal that readily binds the leukocyte in situ (fig.3). This method requires higher affinity nanoparticles to leukocyte and more convenient for clinical settings. Intravital microscopy are often employ to image nanoparticles uptake [24].

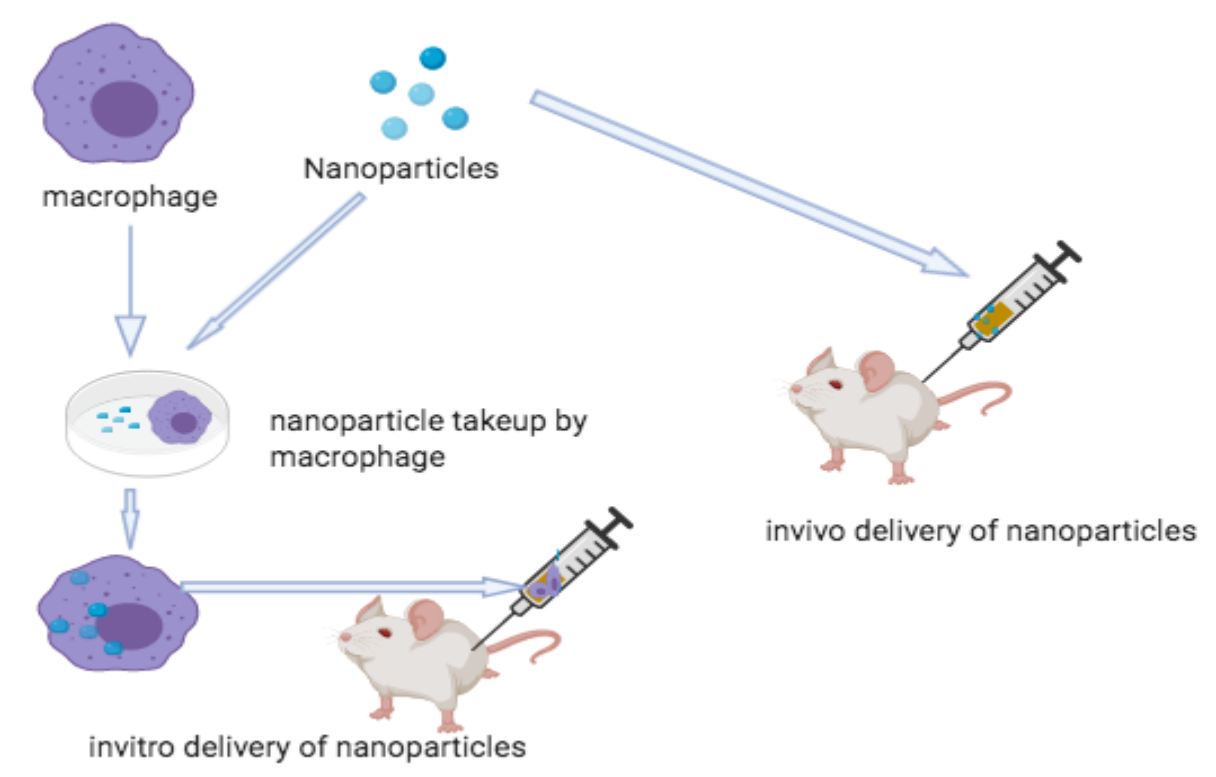

Figure 3: Engineering of macrophage to deliver drug 


\section{Conclusion:}

A combination of diagnostic and therapy are normally directed to the patients to provide tailored therapeutics via the functions in one framework using nanotechnology, that is highly appealing. several nanoparticles found to offer advantages in this technique include polymeric micelles, gold nanoparticles, dendrimers, carbon nanotubes, mesoporous silica nanoparticles, magnetic nanoparticles, up-conversion nanoparticles, polymeric nanoparticles, quantum dots, and solid lipid nanoparticles. Nanotheranostic plays important role in diseases diagnostic, therapy, imaging, monitoring of disease progression / response through the use of nanocarriers. This method is highly applicable to many different types of cancers. 


\section{Reference}

1. Jeelani, S.; Reddy, R.C.; Maheswaran, T.; Asokan, G.S.; Dany, A.; Anand, B. Theranostics: A treasured tailor for tomorrow. J. Pharm. Bioallied Sci. 2014, 6(Suppl 1), S6-S8.

2. Kim, T.H.; Lee, S.; Chen, X. Nanotheranostics for personalized medicine. Expert Rev. Mol. Diagn. 2013, 13, 257-269.

3. Vogenberg, F.R.; Isaacson, B.C.; Pursel, M. Personalized medicine: part 1: evolution and development into theranostics. P T. 2010, 35, 560-576.

4. Tiwari, G.; Tiwari, R.; Sriwastawa, B. et al. Drug delivery systems: An updated review. Int. J. Pharm. Investig. 2012, 2, 2-11.

5. Mango, L. Theranostics: A Unique Concept to Nuclear Medicine. Arch Cancer Sci. Ther. 2017, 1, 001-004.

6. Hacker, M.; Beyer, T.; Baum, R.P.; Kalemis, A.; Lammertsma, A.A.; et al. Nuclear medicine innovations help (drive) healthcare (benefits). Eur. J. Nucl. Med. Mol. Imaging 2015, 42, 173-175.

7. Mango, L.; Pacilio, M. Therapy with Alpha Rays. ARC Journal of Radiology and Medical Imaging. 2016, 1, 1-3.

8. Pacilio, M.; Ventroni, G.; Basile, C.; Ialongo, P.; Becci, D.; et al. Improving the dose-myelotoxicity correlation in radiometabolic therapy of bone metastases with 153Sm-EDTMP. Eur. J. Nucl. Med. Mol. Imaging 2014, 41, 238-252.

9. Goldenberg, DM.; Preston, D.F.; Primus, F.J.; Hansen, H.J.; Photoscan localization of GW-39 tumors in hamsters using radiolabeled anticarcinoembryonic antigen immunoglobulin G. Cancer Res. 1974, 34, 1-9. 
10. Goldenberg, D.M.; DeLand, F.; Kim, E.; Bennett, S.; Primus, F.J. et al. Use of radiolabeled antibodies to carcinoembryonic antigen for the detection and localization of diverse cancers by external photoscanning. N. Engl. J. Med. 1978; 298: 1384-1386

11. Donya, M.; Radford, M.; ElGuindy, A.; Firmin, D.; Yacoub, M.H. Radiation in medicine: Origins, risks and aspirations. Glob. Cardiol. Sci. Pract. 2014, 4, $437-448$.

12. Xie, J.; Lee, S.; Chen, X. Nanoparticle-based theranostic agents. Adv. Drug Deliv. Rev. 2010,62, 1064-1079.

13. Wicki, A.; Witzigmann, D.; Balasubramanian, V.; Huwyler, J. Nanomedicine in cancer therapy: challenges, opportunities, and clinical applications. J. Control Release 2015, 200,138-57.

14. Byrne, J.D.; Betancourt, T.; Brannon-Peppas, L. Active targeting schemes for nanoparticle systems in cancer therapeutics, Adv. Drug Deliv. Rev. 2008,60, $1615-1626$.

15. Maruyama, K.; Takahashi, N.; Tagawa, T.; Nagaike, K.; Iwatsuru, M. Immunoliposomes bearing polyethyleneglycol-coupled Fab' fragment show prolonged circulation time and high extravasation into targeted solid tumors in vivo, FEBS Lett.1997, 413, 177-180.

16. Kamaly, N.; Xiao, Z.; Valencia, P.M.; Radovic-Moreno, A.F.; Farokhzad, O.C. Targeted polymeric therapeutic nanoparticles: design, development and clinical translation. Chem. Soc. Rev.2012, 41, 2971-3010.

17. Huang, H. C.; Barua S.; Sharma, G.; Dey, S.K.; Rege, K.; Inorganic nanoparticles for cancer imaging and therapy. J. Control. Release 2011,155, 
$344-357$.

18. Maggiorella, L.; Barouch, G.; Devaux, C.; Pottier, A.; Deutsch, E.; Bourhis, J. et al. Nanoscale radiotherapy with hafnium oxide nanoparticles, Future Oncol. 2012, 8, 1167-1181.

19. Libutti, S.K.; Paciotti, G.F.; Byrnes, A.A.; Alexander, H.R.; Gannon, W.E.; Walker, M.; et al., Phase I and pharmacokinetic studies of CYT-6091, a novel PEGylated colloidal gold-rhTNF nanomedicine. Clin. Cancer Res. 2010, 16, 6139-6149.

20. Kovalainen, M.; Mönkäre, J.; Kaasalainen, M.; Riikonen, J.; Lehto, V-P.; Salonen, J. et al., Development of porous silicon nanocarriers for parenteral peptide delivery. Mol. Pharm. 2013,10, 353-359.

21. Duncan, R. Polymer conjugates as anticancer nanomedicines. Nat. Rev. Cancer 2006, 6, 688-701.

22. Hawe, A.; Hulse, W.L.; Jiskoot, W.; Forbes, R.T. Taylor dispersion analysis compared to dynamic light scattering for the size analysis of therapeutic peptides and proteins and their aggregates. Pharm. Res. 2011, 28, 2302-2310.

23. 33. Kirn, D.H.; Thorne, S.H. Targeted and armed oncolytic poxviruses: a novel multimechanistic therapeutic class for cancer. Nat. Rev. Cancer 2009, 9, $64-71$.

24. Dong X, Chu D, Wang Z. Leukocyte-mediated Delivery of Nanotherapeutics in Inflammatory and Tumor Sites. Theranostics 2017, 7, 751-763.

25. Irvine, D.J.; Hanson, M.C.; Rakhra, K.; Tokatlian, T. Synthetic Nanoparticles for Vaccines and Immunotherapy. Chem. Rev. 2015; 115:11109-46. 
26. Anselmo, AC.; Gilbert, J.B.; Kumar, S.; Gupta, V.; Cohen, R.E.; Rubner, M.F. et al. Monocyte-mediated delivery of polymeric backpacks to inflamed tissues: a generalized strategy to deliver drugs to treat inflammation. J. Control. Release: 2015,199, 29-36.

27. Stephan, M.T.; Moon, J.J.; Um, S.H.; Bershteyn, A.; Irvine, D.J. Therapeutic cell engineering with surface-conjugated synthetic nanoparticles. Nat. Med. 2010,16, 1035-41. 